\title{
Uniaxial Magnetic Anistotropy of a NiO-Spin Valve Device
}

\author{
Won-Hyung Lee ${ }^{1}$, Do-Guwn Hwang ${ }^{2}$, and Sang-Suk Lee ${ }^{2 *}$ \\ ${ }^{1}$ Department of Electrical Engineering, Freshman, The Pennsylvania State University, PA 16802, U.S.A. \\ ${ }^{2}$ Department of Oriental Biomedical Engineering, Sangii University, Wonju 220-702, Korea
}

(Received 28 January 2009, Received in final form 18 March 2009, Accepted 19 March 2009)

\begin{abstract}
The shape anisotropy effect of a giant magnetoresistance-spin valves (GMR-SV) device with a glass/NiO/NiFe/ $\mathrm{CoFe} / \mathrm{Cu} / \mathrm{CoFe} / \mathrm{NiFe}$ layered structure for use in the detection of magnetic property of molecules within a cell was investigated. The patterned device was given uniaxial anisotropy during the sputtering deposition and vacuum post-annealing, which was performed at $200^{\circ} \mathrm{C}$ under a 300 Oe magnetic field. The pattern size of the device, which was prepared through the photolithography process, was $2 \times 15 \mu \mathrm{m}^{2}$. The experimental results confirmed that the best design for a GMR-SV device to be used as a biosensor is to have both the axis sensing current and the easy axis of the pinned $\mathrm{NiO} / \mathrm{NiFe} / \mathrm{CoFe}$ triple layer oriented in the direction of the device's width, while the easy axis of the free $\mathrm{CoFe} / \mathrm{NiFe}$ bilayer should be pointed along the long axis of the device.
\end{abstract}

Keywords : giant magnetoresistance-spin valve (GMR-SV), shape anisotropy effect, magnetic sensitivity, magnetic easy axis

\section{Introduction}

With the development of nanoengineering technology and its fusion with the biological and medical sciences, giant magnetoresiatance spin valve (GMR-SV) devices have begun to be used in molecular chips based on deoxyribonucleic acid (DNA) [1-5]. The typical biosensor that targets biomolecules for the detection of biosignals consists of a kernel device in combination with the biological recognition system and a physical and chemical transducer, and it can be used in detecting particular molecules [6-8].

Recently there have been attempts using a highly sensitive nano-magnetic biosensor to investigate and observe the magnetic properties of biomolecules. A medical biochip sensor used by several research groups can detect less than a few tens of molecules in the laboratory by using DNA cognitive tag GMR $[9,10]$. Because of the demand for biosensors in the medical and diagnostic fields, this area is thought to have a large potential market. Additionally, optical microscopes can be used to observe the coherent motion of biomolecules inside biotubes. Magnetic instruments can be used to detect their magnetic characteristics.

\footnotetext{
*Corresponding author: Tel: +82-33-738-7961

Fax: +82-33-738-7962, e-mail: sslee@sangji.ac.kr
}

Biosensors that respond to magnetic signals make it easy to observe the particle motion of biological and chemical molecular units. There are reports that the magnetic field variations caused by the motion of nanomagnetic particles of a few $\mu \mathrm{m}$ in size or magnetic beads $10 \mathrm{~nm}$ in size provide enough of a signal to be observed by such devices $[8,9]$.

The selective magnetic sensor device includes biomagnetic materials, such as magnetic beads coated with Streptavidin particles and biotin particles whose superior properties can be enough to make a sufficiently effective nano-bio device in the future [11]. Presently, the study of the detailed response and behavior of biomolecules and cells attached to magnetic beads or nano-particles is a very important factor in the biological and medical sciences.

In our study, we have investigated a method to enhance the sensitivity of nano-magnetic sensitive GMR-SV biosensors. That is, the variation in magnetic properties of GMR-SV biosensor was observed and analyzed, which the magnetic pinned layer and free layer was induced by the magnetic shape anisotropy to the direction of width and length, respectively 


\section{Experimental Method}

On a substrate of Corning glass (\#7059) we prepared GMR-SV biosensors consisting of $\mathrm{NiO}(300 \AA) / \mathrm{NiFe}(20$ $\AA) / \mathrm{CoFe} \quad(10 \AA) / \mathrm{Cu} \quad(26 \AA) / \mathrm{CoFe} \quad(10 \AA) / \mathrm{NiFe} \quad(40 \AA)$ multilayers, which were deposited by RF and DC magnetron sputtering $[12,13]$. The base pressure and working pressure of the argon-gas vacuum chamber were $3 \times 10^{-6}$ Torr and $3 \times 10^{-3}$ Torr, respectively. The distance between the 3-inch target and the sample was about $80 \mathrm{~mm}$. The $\mathrm{RF}$ power during deposition of $\mathrm{NiO}$ was $100 \mathrm{~W}$. The DC applied voltage and current corresponding to the deposition rate and uniformity were $400 \mathrm{~V} / 120 \mathrm{~mA}, 350 \mathrm{~V} / 80$ $\mathrm{mA}$, and $300 \mathrm{~V} / 51 \mathrm{~mA}$ for the deposition of the $\mathrm{NiO}$, $\mathrm{NiFe}, \mathrm{CoFe}$, and $\mathrm{Cu}$, respectively. Under these conditions, the deposition rates of the four targets $\left(\mathrm{NiO}, \mathrm{Ni}_{80} \mathrm{Fe}_{20}\right.$, $\mathrm{Co}_{90} \mathrm{Fe}_{10}$, and $\mathrm{Cu}$ ) were $0.5 \AA / \mathrm{s}, 2.0 \AA / \mathrm{s}, 1.5 \AA / \mathrm{s}$, and 2.0 $\AA / s$, respectively. The uniaxial anisotropic direction was created with an external magnetic field of magnitude approximately 300 Oe.

We first investigated the magnetoresistances property of the four-probe GMR-SV multilayer, which had a width of $8 \mathrm{~mm}$ and a length of $20 \mathrm{~mm}$ and had been prepared by a shadow mask at room temperature. By the lithography process, the sample etched by electron cyclotron resonance (ECR) Ar-ion milling was patterned in the $2 \times 15 \mu \mathrm{m}^{2}$ photoresist (PR) region. The direction of the uniaxial anisotropy of the GMR-SV multilayer was parallel or anti-parallel to the width axis of the device. As shown in Fig. 1, the micro-patterning device with the real region of $2 \times 5 \mu \mathrm{m}^{2}$ after the PR removal by acetone was formed the two-probe $\mathrm{Al}(500 \AA) / \mathrm{Cu}(200 \AA)$ electrode through the lift-off method under the lithography process. The deposition of the $\mathrm{Al} / \mathrm{Cu}$ electrode was done in-situ in order

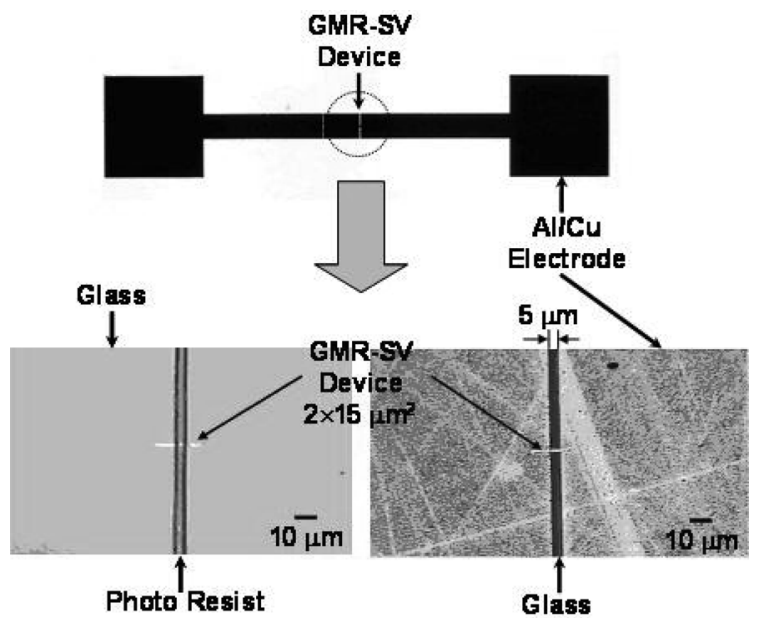

Fig. 1. Schematic and real device features of the fabricated GMR-SV biosensor, with a size of $2 \times 15 \mu \mathrm{m}^{2}$. to minimize changes in the magnetoresistance properties caused by process or the oxidation layer and surface contamination.

To increase the magnetic sensitivity of the GMR-SV biosensor, we subjected the fabricated sample to vacuum annealing under an external 300 Oe magnetic field. During the vacuum annealing the chamber was maintained at a working pressure of $3 \times 10^{-6}$ Torr. During the annealing the temperature was first increased to $200^{\circ} \mathrm{C}$ at a rate of $5{ }^{\circ} \mathrm{C} / \mathrm{min}$, then it was maintained at $200^{\circ} \mathrm{C}$ for $1 \mathrm{hr}$, and, finally, it was lowered back down to room temperature at a rate of $5{ }^{\circ} \mathrm{C} / \mathrm{min}$. The easy axis of the bottom-pinned $\mathrm{CoFe} / \mathrm{NiFe}$ bilayer of GMR-SV device was induced during the annealing process by an external magnetic field applied perpendicular to the device's long axis direction. By contrast, the easy axis of the top free $\mathrm{CoFe} / \mathrm{NiFe}$ bilayer of the GMR-SV device was induced in a direction along the device's long axis.

The coercivity $\left(H_{\mathrm{c}}\right)$, exchange coupling field $\left(H_{\mathrm{ex}}\right)$, magnetoresistance ratio (MR (\%)), and magnetic sensitivity $(M S)$ of the sample and device were measured at room temperatyre by the two- or four-probe method.

\section{Results and Discussion}

Fig. 2 shows the measurements of the magnetoresistance of the GMR-SV multilayer. Before the micropatterning, the glass sample (7059)/ $\mathrm{NiO}(300 \AA) / \mathrm{NiFe}(20$ $\AA) / \mathrm{CoFe}(10 \AA) / \mathrm{Cu}(26 \AA) / \mathrm{CoFe}(10 \AA) / \mathrm{NiFe}(40 \AA)$ had been fabricated using the shadow mask shown in the inset of Fig. 2. Fig. 2(a) and (b) are the major and minor loops of the magnetoresistance (MR) curves as measured by the

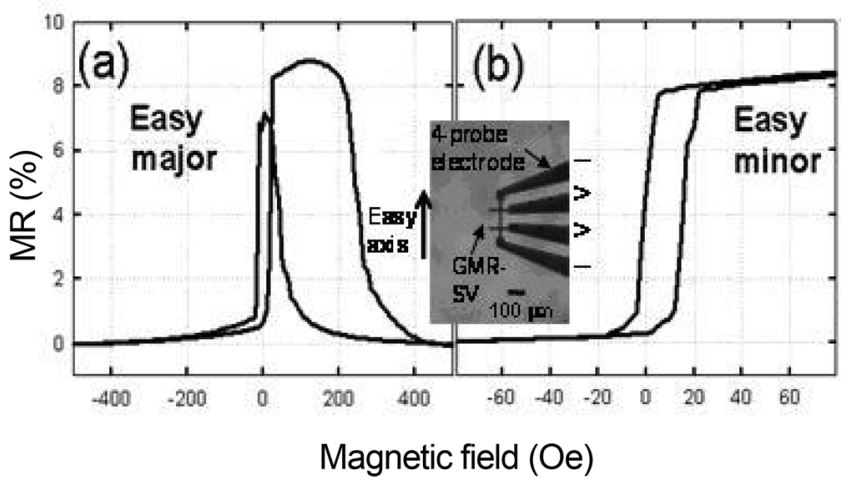

Fig. 2. Magnetoresistive curves measured by the four-probe method for the micro-pre-patterned $\mathrm{NiO}(300 \AA) / \mathrm{NiFe}(20 \AA) /$ $\mathrm{CoFe}(10 \AA) / \mathrm{Cu}(26 \AA) / \mathrm{CoFe}(10 \AA) / \mathrm{NiFe}(40 \AA)$ GMR-SV multilayer structure. The inset shows the patterning size of the shadow mask. (a) The major loop of the MR curve along the easy axis and (b) the minor loop of the MR curve along the easy axis. 
four-probe electrode. The MR ratio and the internal resistance were found to be $8.8 \%$ and $25 \Omega$ as shown in Fig. 2(a). The pinned layer, which was a $\mathrm{NiO}(300 \AA) /$ $\mathrm{NiFe}(20 \AA) / \mathrm{CoFe}(10 \AA)$ trilayer, had $H_{\mathrm{ex}}$ and $H_{\mathrm{c}}$ of 140 $\mathrm{Oe}$ and $105 \mathrm{Oe}$, respectively. The $\mathrm{CoFe}(10 \AA) / \mathrm{NiFe}$ (40 $\AA$ ) bilayer of the free layer above the nonmagnetic $\mathrm{Cu}$ layer was measured to have an interlayer coupling field and a coercivity between the two bottom-pinned layers and the top free layer were of $8.0 \mathrm{Oe}$ and $8.2 \mathrm{Oe}$, respectively, as shown in Fig. 2(b). Finally, the external magnetic sensitivity $(M S)$, which determines the magnetic characteristics of a highly sensitive device, was found to be about $1.5 \% / \mathrm{Oe}$. This demonstrated that the sample could be expected to maintain good magnetic sensitivity after the micro-patterning. When the magnetic field was applied to the hard axis of the GMR-SV multilayer, the MR ratio was found to be about $2.5 \%$, with the decrease caused by the anisotropic magnetoresistance (AMR) effect.

Fig. 3 shows the magnetoresistive curves for the NiObased GMR-SV multilayer biosensor, which were measured after the micro-patterning process by the two-probe method. The inset of Fig. 3 shows the $2 \times 5 \mu \mathrm{m}^{2}$ micropatterned feature except the part coated with the $\mathrm{Al} / \mathrm{Cu}$ electrode along an arrow indicating the easy axis. The measured direction of the easy axis was corresponded to uniaxial anisotropy during the deposition. The external field was applied parallel to the easy axis and sensing current. As shown in Fig. 3(a) and 3(b), the properties of the major loop of the magnetoresistive curve measured along the easy axis differed from those shown of Fig. 2(a), in which the data were taken before the patterning process. In particular, the values of $H_{\mathrm{ex}}$ and $H_{\mathrm{c}}$ were decreased slightly, to 120 Oe and $90 \mathrm{Oe}$, respectively. On the other hand, the MR and $M S$ values were decreased sharply, to $4.2 \%$ and $0.3 \% / \mathrm{Oe}$, respectively. Fig. 3(b) shows that the intensity of interlayer coupling of between the bottom-pinned $\mathrm{NiFe} / \mathrm{CoFe}$ layer and the top free $\mathrm{CoFe} / \mathrm{NiFe}$ layer was about $8.0 \mathrm{Oe}$, which was similar to what was measured in one of the pre-patterned devices. As though the effect caused by the difference between the initial direction of the magnetization configuration and the direction of the measuring current, it seems was larger than the effect of the demagnetization (shape anisotropy). These phenomena show that not only did the resistance of the two probe electrodes, including surface resistance, increased by up to $85 \Omega$, but also the soft $\left(90^{\circ} \mathrm{C}\right)$ and hard baking $\left(110^{\circ} \mathrm{C}\right)$ during the lithography process caused the thermal effect and the surface contamination of the free layer.

To transform the direction of the easy axis of the NiObased GMR-SV multilayer biosensor from pointing along

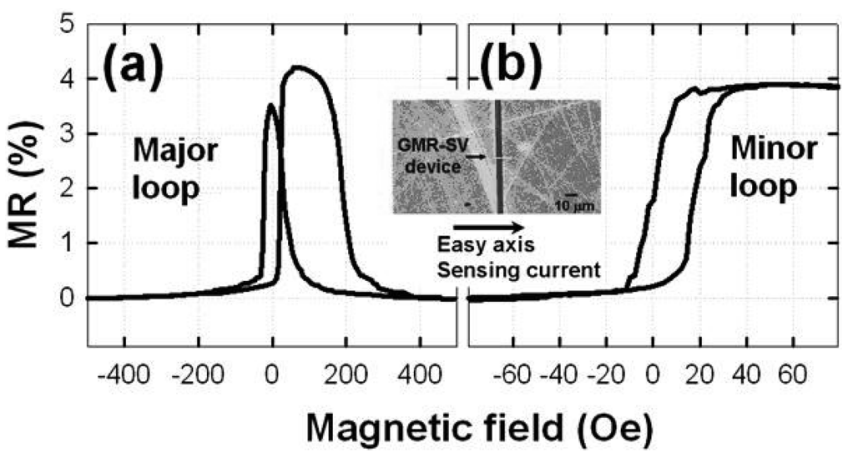

Fig. 3. Magnetoresistive curves measured by the two-probe method for the micro-post-patterned $\mathrm{NiO}(300 \AA) / \mathrm{NiFe}(20$ $\AA) / \mathrm{CoFe}(10 \AA \mathrm{p}) / \mathrm{Cu}(26 \AA) / \mathrm{CoFe}(10 \AA) / \mathrm{NiFe}(40 \AA) \mathrm{GMR}-$ $\mathrm{SV}$ multilayer structure: Inset shows the micro-patterned feature, which had a size of $2 \times 15 \mu^{2}$, and the direction of the easy axis. (a) The major loop of the MR curve along the easy axis and (b) the minor loop of the MR curve along the easy axis.

the length of the device, as was the case in Fig. 3, to pointing transversally across the device, we performed vacuum thermal annealing at $200{ }^{\circ} \mathrm{C}$ for $1 \mathrm{hr}$ under a uniform magnetic field provided by a permanent 300 Oe magnet. The inset of Fig. 4 shows the micro-patterned $2 \times 15 \mu \mathrm{m}^{2}$ device, the directions of the easy and hard axes, and the direction of the sensing current along the hard axis. After the thermal annealing process, we measured the major and minor loops of the magnetoresistive curves along the easy axis using the two-probe method at room temperature; the results are shown in Fig. 4(a) and 4(b).

The values of $H_{\mathrm{ex}}, H_{\mathrm{c}}$, and MR as measured from the magnetoresistive curves were about $120 \mathrm{Oe}, 90 \mathrm{Oe}$, and $4 \%$, respectively, both before and after the annealing process. By contrast, the value of $M S$ was lower after thermal annealing by a factor of three, dropping from $0.3 \% /$ Oe to $0.1 \% /$ Oe. Before annealing, the easy axis minor loop showed an $H_{\mathrm{c}}$ of 20 Oe. After annealing, the intensity of the interlayer coupling changed to 2 Oe. As the case with the MR curves shown in Fig. 3(a) and (b), the effect by the difference between initial magnetization direction and measuring the current direction was larger than the demagnetization effect. From this result, the final MR curve shows linear property for a slightly external magnetic field on the neighboring region of $0 \mathrm{Oe}$ after the annealing process. The enhanced minor MR loop can be used by the biosensor in the detection of magnetic nanoparticles.

By using vacuum thermal annealing under the magnetic field with uniaxial anisotropy, one can induce the easy axis of the magnetic pinned layer of the GMR-SV bio- 


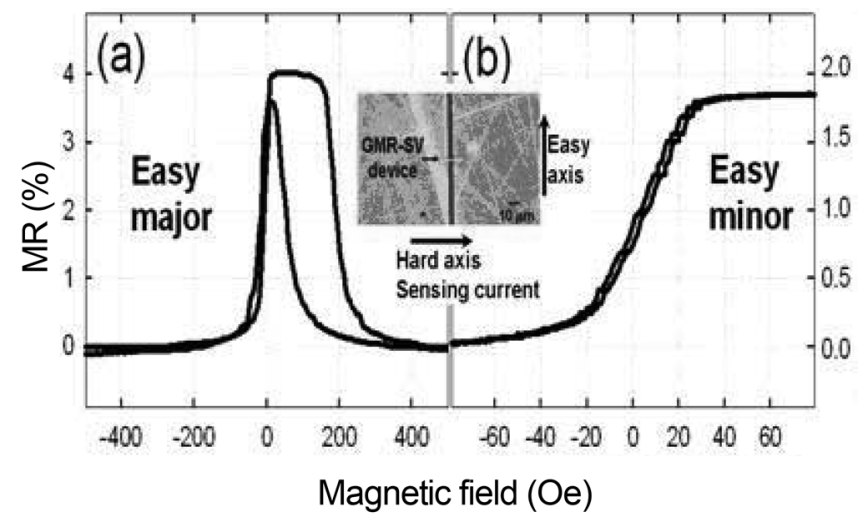

Fig. 4. Magnetoresistive curves measured by the two-probe method for the micro-post-patterned and post-annealed $\mathrm{NiO}$ $(300 \AA) / \mathrm{NiFe}(20 \AA) / \mathrm{CoFe}(10 \AA) / \mathrm{Cu} \quad(26 \AA) / \mathrm{CoFe}(10 \AA) /$ $\mathrm{NiFe}(40 \AA)$ GMR-SV multilayer structure. The inset shows the micro-patterning $2 \times 15 \mu^{2}$ device and the directions of the easy axis and hard axis. (a) The major loop of the MR curve along the easy axis and (b) the minor loop of the MR curve along the easy axis.

sensor to lie along the "width direction," that is, the direction perpendicular to the length of the device. Meanwhile, shape magnetic anisotropy can be used to induce the easy axis of the magnetic free layer to lie along the long axis. This shows that our GMR-SV biosensor is sufficient for its purposes. That is, the easy axis formed by the applied magnetic field of uniaxial anisotropy during the deposition of GMR-SV multilayer was very important to pattern the micro device perpendicular to the length direction of the sample. In this study, although the magnetic sensitivity was decreased, the reason to perform the shape anisotropy through the thermal annealing process was to obtain the optimum condition to minimize and respond to the coercivity of the GMR-SV biosensor in the surrounding region of 0 Oe by a subtle external magnetic field with increase and decrease. When the GMR-SV device is used as a biosensor with an ultramicro magnetic field, sufficient magnetic sensitivity and large output amplitudes are important characteristics. Therefore, we prepared the device so that the uniaxial magnetic anisotropies of the magnetic pinned layer and the very soft magnetic free layer were orthogonal to each other. The magnetoresistance property of the micropatterned device has linear sensitivity and a GMR effect caused by the AMR effect and the large MR amplitude. On the other hand, for all of the devices put through the vacuum annealing treatment, we need to eliminate the fluctuation of the easy axis at $150{ }^{\circ} \mathrm{C}$ under the lithography process. Furthermore, for GMR-SV devices based on as-grown IrMn, FeMn, and NiMn, our experimental results suggest that the post-annealing treatment in the final stage needs to the establishment of the easy axis of pinned layer and the shape magnetic anisotropy of free layer.

\section{Conclusion}

We investigated a GMR-SV device with a glass $/ \mathrm{NiO} /$ $\mathrm{NiFe} / \mathrm{CoFe} / \mathrm{Cu} / \mathrm{CoFe} / \mathrm{NiFe}$ structure for use in detecting the magnetic properties of molecules inside the cell. The magnetoresistance of the prepared device depends on creating two features that have easy axes perpendicular to one another, with one longitudinal and the other transverse. The patterned device was given with a uniaxial anisotropy direction during its deposition by sputtering and vacuum post-annealing at $200{ }^{\circ} \mathrm{C}$ under a magnetic field with magnitude 300 Oe. Taking into account the magnetic shape anisotropy effect, we decided to make the micro-patterned device $2 \times 15 \mu \mathrm{m}^{2}$ in size after the photo lithography process. Our experimental results confirmed that the best design for a GMR-SV device to be used as a biosensor is to have both the axis sensing current and the easy axis of the pinned $\mathrm{NiO} / \mathrm{NiFe} / \mathrm{CoFe}$ triple layer oriented in the direction of the device's width, while the easy axis of the free $\mathrm{CoFe} / \mathrm{NiFe}$ bilayer should be pointed along the long axis of the device.

\section{Acknowledgment}

This work was supported in part by the Korea Foundation for International Cooperation of Science \& Technology (KICOS K20702000015-07E0200-0510) and the Sangji University Research Program (2007-2008).

\section{References}

[1] D. R. Baselt, G. U. Lee, M. Natesan, S. W. Metzger, P. E. Sheehan, and R. J. Colton, Biosens. Bioelectron. 13, 731 (1998).

[2] P. Kollu, D. Y. Kim, and C. G. Kim, J. Magnetics 12, 35 (2007).

[3] G. Li, S. Sun, R. J. Wilson, R. L. White, N. Pourmand, and S. X. Wang, Sens. Acut. A126, 98 (2006); G. Li and S. X. Wang, IEEE Trans. Magn. 39, 3313 (2003).

[4] S. H. Park, K. S. Soh, D. G. Hwang, J. R. Rhee, and S. S. Lee, J. Magnetics 13, 30 (2008).

[5] D. L. Graham, H. A. Ferreira, P. P. Fretias, and J. M. S. Cabral, Biosens. Bioelectron. 18, 483 (2003).

[6] J. S. In, S. H. Kim, J. Y. Kang, A. Tiwari, and J. I. Hong, J. Magnetics 12, 118 (2007).

[7] S. H. Park, K. S. Soh, M. C. Ahn, D. G. Hwang, and S. S. Lee, J. Kor. Mag. Soc. (Korean) 16, 157 (2006). 
[8] S. S. Lee, S. H. Park, and K. S. Soh, Sae Mulli (Korean) 52, 564(2006).

[9] B. M. de Boer, J. A. H. M. Kahlman, T. P. G. H. Jansen, H. Duric, and J. Veen, Biosens. Bioelectron. 22, 2366 (2006).

[10] S. X. Wang, S.-Y Bae, G. Li, S. Sun, R. L. White, J. T. Kemp, and C. D. Webb, J. Magn. Magn. Mater. 293, 731
(2005).

[11] S. S. Lee, D. G. Hwang, J. K. Kim, and K. Rhie, J. Kor. Phys. Soc. 40, 484 (2002).

[12] G. Choi, S. S. Lee, D. G. Hwang, and S. W. Kim, Sae Mulli (Korean) 50, 334 (2005).

[13] K. Y. Kim, H. C. Choi, C. Y. You, and J. S. Lee, J. Magnetics 13, 97 (2008). 